\title{
A low-frequency noise model with carrier generation-recombination process for pentacene organic thin-film transistor
}

Cite as: J. Appl. Phys. 114, 044503 (2013); https://doi.org/10.1063/1.4816103

Submitted: 09 March 2013 . Accepted: 03 July 2013 . Published Online: 22 July 2013

C. Y. Han, L. X. Qian, C. H. Leung, C. M. Che, and P. T. Lai
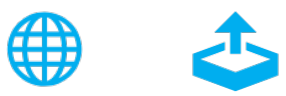

View Online

Export Citation

\section{ARTICLES YOU MAY BE INTERESTED IN}

Correlation between microstructure, electronic properties and flicker noise in organic thin film transistors

Applied Physics Letters 92, 132103 (2008); https://doi.org/10.1063/1.2903508

$1 / \mathrm{f}$ noise in pentacene organic thin film transistors

Journal of Applied Physics 88, 5395 (2000); https://doi.org/10.1063/1.1314618

Measurement, analysis, and modeling of $1 / \mathrm{f}$ noise in pentacene thin film transistors

Applied Physics Letters 99, 062106 (2011); https://doi.org/10.1063/1.3622651 


\title{
A low-frequency noise model with carrier generation-recombination process for pentacene organic thin-film transistor
}

\author{
C. Y. Han, ${ }^{1}$ L. X. Qian, ${ }^{1}$ C. H. Leung, ${ }^{1}$ C. M. Che ${ }^{2}$ and P. T. Lai ${ }^{1, a)}$ \\ ${ }^{1}$ Department of Electrical and Electronic Engineering, The University of Hong Kong, Pokfulam Road, \\ Hong Kong \\ ${ }^{2}$ Department of Chemistry, The University of Hong Kong, Pokfulam Road, Hong Kong
}

(Received 9 March 2013; accepted 3 July 2013; published online 22 July 2013)

\begin{abstract}
By including the generation-recombination process of charge carriers in conduction channel, a model for low-frequency noise in pentacene organic thin-film transistors (OTFTs) is proposed. In this model, the slope and magnitude of power spectral density for low-frequency noise are related to the traps in the gate dielectric and accumulation layer of the OTFT for the first time. The model can well fit the measured low-frequency noise data of pentacene OTFTs with $\mathrm{HfO}_{2}$ or $\mathrm{HfLaO}_{\text {gate }}$ dielectric, which validates this model, thus providing an estimate on the densities of traps in the gate dielectric and accumulation layer. It is revealed that the traps in the accumulation layer are much more than those in the gate dielectric, and so dominate the low-frequency noise of pentacene OTFTs. (C) 2013 AIP Publishing LLC. [http://dx.doi.org/10.1063/1.4816103]
\end{abstract}

\section{INTRODUCTION}

Organic thin-film transistors (OTFTs) have gained considerable interest due to their potential application in large-area flexible displays and sensor arrays. ${ }^{1,2}$ Among various organic semiconductors, pentacene has higher carrier mobility than others, reaching well above $1 \mathrm{~cm}^{2} / \mathrm{V} \cdot \mathrm{s} .^{3,4}$ Hence, it has been widely employed as the active layer in the OTFTs. Flicker noise, also called $1 / f$ noise because its power spectral density (PSD) varies as $1 / f^{\alpha}$ with $\alpha$ close or equal to unity, occurs in almost all semiconductor devices in the lowfrequency region. ${ }^{5}$ In the last several decades, the flicker noise in the metal-oxide-semiconductor field-effect transistors (MOSFETs) has been intensively studied. ${ }^{6-8}$ For the flicker noise in the silicon MOSFET, the exponent $\alpha$ is close to one in most cases. Assuming the probability of an electron penetrating into the oxide decreases exponentially with the distance from the oxide-semiconductor interface and the traps are uniformly or exponentially distributed in the oxide, the value of the slope of the PSD of the low-frequency noise (LFN) of MOSFETs can be explained..$^{9-11}$ It is clear that pentacene OTFTs are quite different from the conventional MOSFET. The carrier mobility of OTFTs is much lower than that of silicon MOSFET. Moreover, there are many traps in the pentacene grains and the grain boundaries of pentacene OTFTs. ${ }^{12,13}$ However, in the flicker noise model for the conventional MOSFET based on single-crystal silicon, traps are assumed to exist only in the oxide near the interface. ${ }^{11,14}$

At present, flicker noise models for the silicon MOSFET are frequently employed to interpret the noise data measured in the pentacene OTFTs. ${ }^{15,16}$ Considering the difference between the OTFT and conventional inorganic MOSFET, it is necessary to bring forward a model for the flicker noise in the OTFTs. Several papers reported that the PSD of LFN in OTFTs significantly deviated from the $1 / f$ noise model for

\footnotetext{
a) Author to whom correspondence should be addressed. Electronic address: laip@eee.hku.hk.
}

the MOSFET. ${ }^{17-20}$ Both the magnitude and slope of the PSD are still not well modeled from the physics foundation. Previous reports have mentioned that the traps in the grain boundaries dominate the LFN in OTFTs and even proposed that the traps in the boundaries could affect the slope of the PSD. ${ }^{15,20,21}$ However, the relation between the slope and the traps in OTFTs is still not yet provided by any model. In this work, we propose a model based on the generationrecombination (GR) process of charge carriers in the accumulation layer and gate dielectric to explain the slope and magnitude of the PSD measured for pentacene OTFTs.

To verify the proposed model, pentacene OTFTs with $\mathrm{HfO}_{2}$ or $\mathrm{HfLaO}$ gate dielectric were fabricated. After silicon substrates were cleaned by the standard RCA method, $\mathrm{HfO}_{2}$ was deposited by atomic layer deposition (ALD) with a thickness of $40.0 \mathrm{~nm}$ at a substrate temperature of $300^{\circ} \mathrm{C}$, while $\mathrm{HfLaO}$ was prepared by sputtering with a thickness of $39.8 \mathrm{~nm}$ at room temperature. Then, only the HfLaO film was annealed in $\mathrm{N}_{2}$ at $400{ }^{\circ} \mathrm{C}$ for $10 \mathrm{~min}$ to achieve higher film quality. After that, a 30-nm pentacene film was deposited at a rate of $1.1 \mathrm{~nm} / \mathrm{min}$ in high vacuum $\left(4 \times 10^{-6}\right.$ torr $)$ on the two dielectrics. Finally, drain and source electrodes were evaporated on the pentacene film by gold evaporation through a shadow mask to form the OTFTs. The width and length of the channel on the shadow mask were $200 \mu \mathrm{m}$ and $30 \mu \mathrm{m}$, respectively. HP 4145B semiconductor parameter analyzer, Berkeley Technology Associates FET Noise Analyzer Model 9603, and HP 35665A Dynamic Signal Analyzer were used to measure the transfer characteristics and noise spectrum of the pentacene OTFTs. All the measurements were conducted at room temperature $(300 \mathrm{~K})$ in air, and under an electrically shielded environment.

\section{DERIVATION OF THE NOISE MODEL}

From Fig. 1 , the elemental volumes, $\Delta \Lambda$ and $\Delta \Lambda^{\prime}$, in the gate dielectric and accumulation layer, respectively, can be written as 


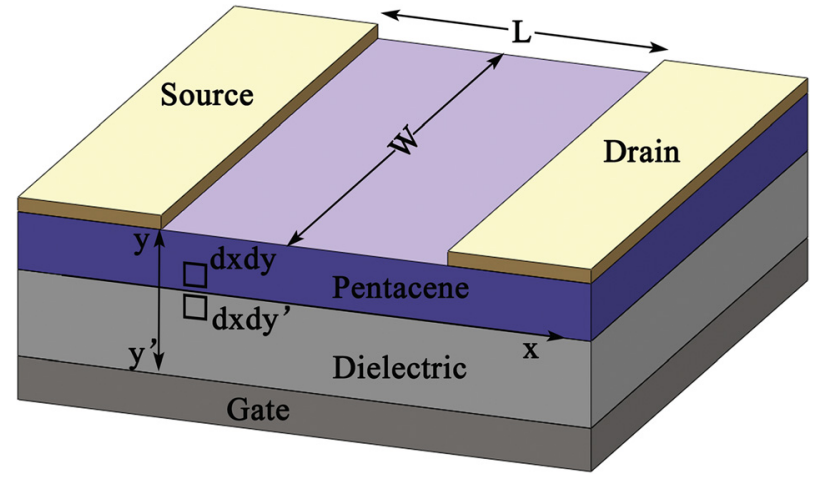

FIG. 1. Structure of the pentacene OTFT used in this study.

$$
\begin{aligned}
\Delta \Lambda & =W d x d y, \\
\Delta \Lambda^{\prime} & =W d x d y^{\prime}
\end{aligned}
$$

where $W$ is the channel width.

Based on the carrier GR process, the frequency spectrum of the mean-squared fluctuation in the number of trapped carriers in an elemental volume is given $b^{22}$

(in the gate dielectric)

$$
S_{\Delta \Lambda \delta \mathrm{n}_{t d}}(f)=\frac{4 \tau_{d}}{1+(2 \pi f)^{2} \tau_{d}^{2}} N_{t d} f_{t d} f_{t d p} \Delta \Lambda,
$$

(in the accumulation layer)

$$
S_{\Delta \Lambda^{\prime} \delta \mathrm{n}_{t b}}(f)=\frac{4 \tau_{b}}{1+(2 \pi f)^{2} \tau_{b}^{2}} N_{t b} f_{t b} f_{t b p} \Delta \Lambda^{\prime},
$$

where

$$
\begin{aligned}
& f_{t d}=1-f_{t d p}=\left[1+\exp \left(E-E_{f d}\right) / k T\right]^{-1} \\
& f_{t b}=1-f_{t b p}=\left[1+\exp \left(E-E_{f b}\right) / k T\right]^{-1}
\end{aligned}
$$

$\delta \mathrm{n}_{\mathrm{td}}$, the fluctuation in trapped carrier density in gate dielectric

$\delta \mathrm{n}_{\mathrm{tb}}$, the fluctuation in trapped carrier density in accumulation layer

$N_{t d}$, the density of traps in gate dielectric

$N_{t b}$, the density of traps in accumulation layer

$k$, the Boltzmann's constant $\left(8.617 \times 10^{-5} \mathrm{eV} \mathrm{K}^{-1}\right)$

$T$, the temperature

$E_{f d}$, the quasi-Fermi level in gate dielectric

$E_{f b}$, the quasi-Fermi level in accumulation layer

$f_{t d}$, the occupancy function in gate dielectric

$f_{t b}$, the occupancy function in accumulation layer

$\tau_{d}$, the decay time in gate dielectric

$\tau_{b}$, the decay time in accumulation layer

Since the fluctuations in carrier number caused by the traps in the gate dielectric and accumulation layer are independent, in the volume $\Delta \Lambda$ and $\Delta \Lambda^{\prime}$, the PSD of the mean-squared fluctuation in current, $\left\langle\left(\delta I_{D}\right)^{2}\right\rangle$, at the point $x$ along the channel can be expressed as (see Appendix A),

$$
\begin{aligned}
S_{\delta I_{D}}(f)= & \frac{4 \mu q^{2} I_{D}}{C_{o x} L^{2}\left|V_{G}-V_{t h}\right|}\left[\frac{\tau_{d}}{1+(2 \pi f)^{2} \tau_{d}^{2}} N_{t d} f_{t d} f_{t d p} d y d V\right. \\
& \left.+\frac{\tau_{b}}{1+(2 \pi f)^{2} \tau_{b}^{2}} N_{t b} f_{t b} f_{t b p} d y^{\prime} d V\right] .
\end{aligned}
$$

The total PSD of the LFN in the drain current is then obtained by integrating the Eq. (3):

$$
\begin{aligned}
S_{I_{D}}(f)= & \frac{4 \mu q^{2} I_{D}}{C_{o x} L^{2}\left|V_{G}-V_{t h}\right|} \\
& \times\left[\int_{0}^{V_{d}} \int_{E_{v}}^{E_{c} \int_{0}^{t_{d}}} \frac{\tau_{d}(E, V, y)}{1+(2 \pi f)^{2} \tau_{d}^{2}(E, V, y)} N_{t d} f_{t d} f_{t d p} d y d E d V\right. \\
& \left.+\int_{0}^{V_{d}} \int_{E_{v}}^{E_{c}} \int_{0}^{t_{b}} \frac{\tau_{b}\left(E, V, y^{\prime}\right)}{1+(2 \pi f)^{2} \tau_{b}^{2}\left(E, V, y^{\prime}\right)} N_{t b} f_{t b} f_{t b p} d y^{\prime} d E d V\right],
\end{aligned}
$$

where $t_{b}$ is the thickness of the accumulation layer $(0.9 \mathrm{~nm}),{ }^{23}$ $t_{d}$ is the distance inside the oxide over which the traps are distributed, and $E_{c}-E_{v}$ is the bandgap energy of pentacene.

For evaluating the integral in Eq. (4), two assumptions have to be made:

(i) The decay time $\tau_{b}$ in the accumulation layer is constant and in the gate dielectric is given by ${ }^{6,24}$ $\tau_{d}(E, V, y)=\tau_{d 0} \exp (\gamma y) \quad$ with $\quad \gamma=4 \pi / h \sqrt{2 m^{*} \Phi_{B}}$, where $\mathrm{m}^{*}$ is the effective hole mass $\left(=3 \mathrm{~m}_{0}{ }^{25}\right), \Phi_{B}$ is the barrier height $(=1.3 \mathrm{eV}), h$ is the Planck's constant, $\tau_{d 0}$ is life time at the interface, and $\gamma$ is the tunneling coefficient $\left(=2 \times 10^{8} \mathrm{~cm}^{-1}\right) .^{10,26}$

(ii) The traps are assumed to be uniformly distributed in the bandgap of pentacene. ${ }^{24}$ With these two assumptions, carrying out the integration in Eq. (4) yields (see Appendix B)

$$
S_{I_{D}}(f)=\frac{A}{f}+\frac{\tau_{b} B}{1+\left(2 \pi f \tau_{b}\right)^{2}},
$$

with

$$
A=\frac{k T V_{d} \mu q^{2} I_{D}}{\gamma C_{o x} L^{2}\left|V_{G}-V_{t h}\right|} N_{t d}
$$

and

$$
B=\frac{4 k T V_{d} \mathrm{t}_{b} \tau_{b} \mu q^{2} I_{D}}{C_{o x} L^{2}\left|V_{G}-V_{t h}\right|} N_{t b} .
$$

The first term in Eq. (5) results from carrier-number fluctuation caused by the traps in the dielectric and is consistent with other noise models based on carrier-number fluctuation for conventional transistors. ${ }^{14}$ On the other hand, the second term refers to the GR noise caused by the traps in the conducting accumulation layer. For the first time, the GR noise caused by the pentacene film has been considered and formulated in the noise model for OTFT. 


\section{RESULTS AND DISCUSSION}

The carrier mobility and threshold voltage of the OTFTs are extracted from their I-V characteristics shown in Fig. 2 and listed in Table I. On the other hand, the method for extracting the noise parameters of the OTFTs is based on the differential evolution algorithm. ${ }^{27}$ The coefficients A, B, and $\tau_{b}$ can be optimally extracted by fitting Eq. (5) with the measured noise spectra in Fig. 3, and their values are listed in Table I. Then, by putting the extracted values of A and B into Eqs. (6) and (7), respectively, $N_{t d}$ and $N_{t b}$ can be calculated and are listed in Table I.

Table I also lists the interface-trap density of the OTFTs $\left(\mathrm{N}_{\mathrm{T}}\right.$ and $\mathrm{N}_{\mathrm{SS}}$ ), where $\mathrm{N}_{\mathrm{T}}$ is extracted by the traditional noise model for silicon MOSFET based on carrier-number fluctuation $^{15}$

$$
\frac{S_{I_{D}}(f)}{I_{D}^{2}}=\frac{g_{m}^{2}}{I_{D}^{2}} \frac{q^{2} k T N_{T}}{W L C_{o x}^{2} f^{\alpha}},
$$

and $\mathrm{N}_{\mathrm{SS}}$ is calculated from the sub-threshold swing (SS) of the OTFTs based on ${ }^{12}$

$$
N_{s s}=\frac{C_{o x}}{q t_{b}}\left[\frac{S S \cdot q}{k T \ln 10}-1\right] .
$$

So, with the same order of magnitude for $\mathrm{N}_{\mathrm{T}}$ and $\mathrm{N}_{\mathrm{SS}}$, the interface-trap density extracted from the traditional noise model is consistent with that extracted from the currentvoltage characteristics of the OTFTs. However, both Eqs. (8) and (9) can only give the effective trap density at the interface because they do not differentiate between the traps in the gate dielectric and accumulation layer.

Unlike the MOSFET based on crystalline silicon, there are many defects in the conducting layer of OTFT. The charge transport mechanisms in organic materials have been described by Coropceanuet et al. ${ }^{28}$ and Lin et al. ${ }^{29}$ The polaron models have been employed to explain the transport mechanism, in which the total carrier mobility can be expressed to a good approximation as a sum of two contributions,

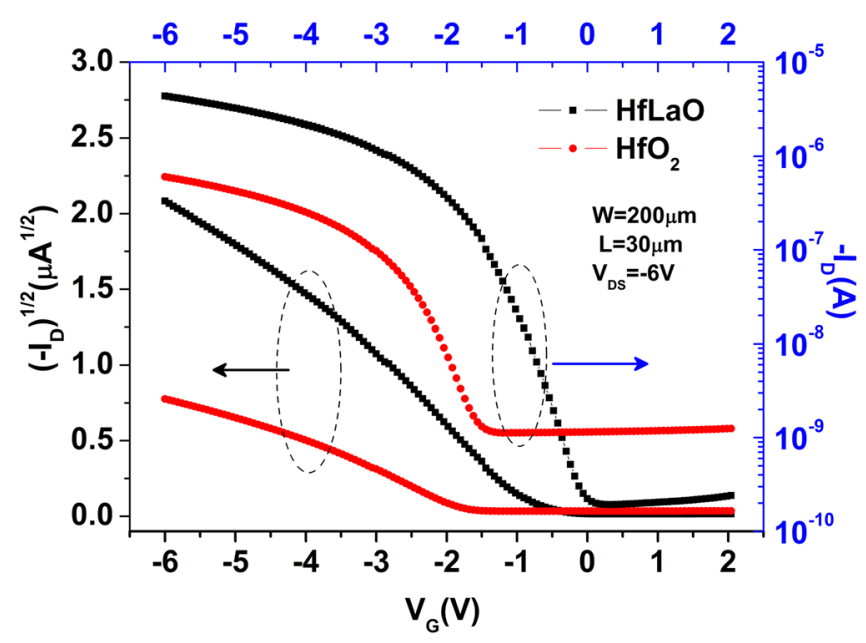

FIG. 2. Transfer characteristics of the OTFTs with sputtered HfLaO and ALD $\mathrm{HfO}_{2}$ as gate dielectric at $\mathrm{V}_{\mathrm{D}}=-5 \mathrm{~V}$.

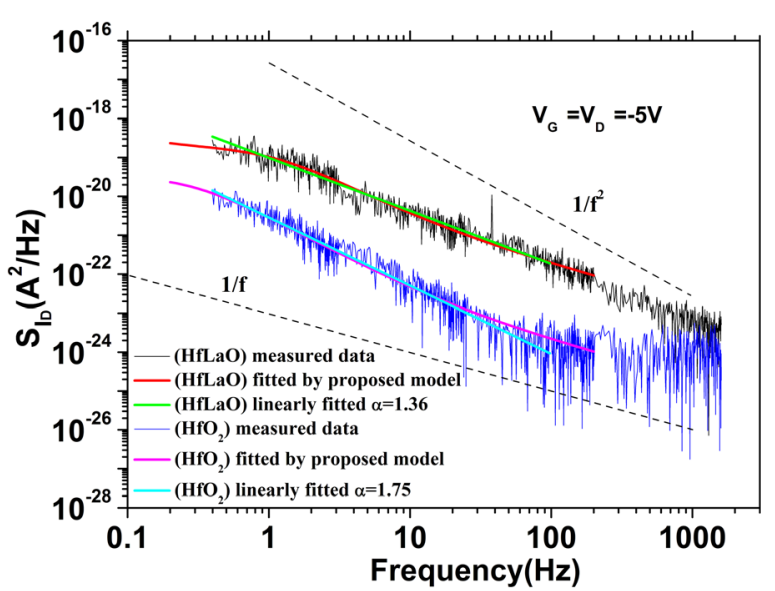

FIG. 3. PSD of drain-current noise for the OTFTs with sputtered HfLaO and ALD $\mathrm{HfO}_{2}$ as gate dielectric. The linearly fitted slope based on a traditional noise model is 1.34 and 1.75 , respectively. On the other hand, the proposed model can better fit the measured data.

$$
\mu=\mu_{t u n}+\mu_{\text {hop }}
$$

where $\mu_{t u n}$ is tunneling mobility and $\mu_{\text {hop }}$ is hopping mobility. At room temperature, the hopping term dominates the mobility, and the mobility exhibits temperature-activated transport. In this study, measurements on the properties of devices are done at room temperature, and thus the hopping conduction should dominate the charge transport in the channel. Therefore, the noise spectrum can be used to quantify the defect density as listed in Table I.

The calculated results by this model are in good agreement with the measured PSD of both OTFTs in the lowfrequency region as shown in Fig. 3. In the high-frequency region (above $100 \mathrm{~Hz}$ for the OTFT with $\mathrm{HfO}_{2}$ gate dielectric and $1000 \mathrm{~Hz}$ for the one with $\mathrm{HfLaO}$ gate dielectric), the low-frequency noise is overshadowed by thermal noise. Based on Eq. (8), the slope of PSD $(\alpha)$ in the low-frequency region can be extracted as 1.75 and 1.34 for the OTFTs with $\mathrm{HfO}_{2}$ and $\mathrm{HfLaO}$ gate dielectric, respectively. However, $\alpha$ is only a fitting parameter without any physical meaning. The proposed model in this work can well fit the slope by including the traps in the accumulation layer, and thus can well explain why the slope significantly deviates

TABLE I. Parameters of the Pentacene OTFTs.

\begin{tabular}{lcc}
\hline \hline Dielectric & $\mathrm{HfLaO}$ & $\mathrm{HfO}_{2}$ \\
\hline Capacitance $C_{o x}\left(\mu \mathrm{F} / \mathrm{cm}^{2}\right)$ & 0.240 & 0.312 \\
Threshold voltage $V_{t h}(\mathrm{~V})$ & -0.587 & -1.85 \\
Drain current $I_{D}(\mu \mathrm{A})\left(\mathrm{V}_{\mathrm{G}}=\mathrm{V}_{\mathrm{D}}=-5 \mathrm{~V}\right)$ & 4.31 & 0.661 \\
Carrier mobility $\mu\left(\mathrm{cm}^{2} / \mathrm{V} \cdot \mathrm{s}\right)$ & 0.358 & 0.0815 \\
Subthreshold swing $(\mathrm{V} / \mathrm{dec})$ & 0.585 & 0.569 \\
$\mathrm{~A}\left(\times 10^{-22} \mathrm{~A}^{2}\right)$ & 36.7 & 0.105 \\
$\mathrm{~B}\left(\times 10^{-20} \mathrm{~A}^{2} \mathrm{~s}^{-1}\right)$ & 31.7 & 4.49 \\
$\tau_{b}(\mathrm{~s})$ & 0.139 & 0.390 \\
$\mathrm{~N}_{\mathrm{td}}\left(\times 10^{20} \mathrm{eV}^{-1} \mathrm{~cm}^{-3}\right)$ & 13.7 & 1.04 \\
$\mathrm{~N}_{\mathrm{tb}}\left(\times 10^{22} \mathrm{eV}^{-1} \mathrm{~cm}^{-3}\right)$ & 1.18 & 6.37 \\
$\mathrm{~N}_{\mathrm{T}}\left(\times 10^{20} \mathrm{eV}^{-1} \mathrm{~cm}^{-3}\right)$ & 3.48 & 6.08 \\
$\mathrm{~N}_{\mathrm{SS}}\left(\times 10^{20} \mathrm{eV}^{-1} \mathrm{~cm}^{-3}\right)$ & 1.85 & 1.47 \\
\hline \hline
\end{tabular}




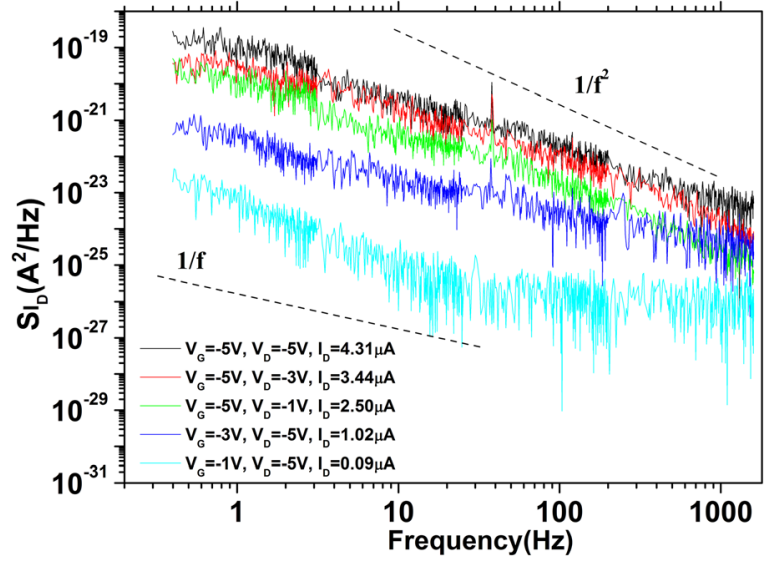

FIG. 4. PSD of drain-current noise for the OTFT with sputtered HfLaO as gate dielectric at various drain biases and gate voltages.

from the value of unity in the $1 / f$ noise model. As shown in Table I, the density of the traps in the accumulation layer is higher than that in the gate dielectric. This can explain why the slope is significantly larger than one and verify that the traps in the pentacene accumulation layer dominate the LFN. The slope is determined by the ratio of the trap densities in the conducting layer and gate dielectric and also the decay time in the conducting layer. Compared with the OTFT with $\mathrm{HfLaO}$ gate dielectric, the one with $\mathrm{HfO}_{2}$ gate dielectric has higher density of traps in the accumulation layer, but lower density of traps in the gate dielectric. This is because the ALD technique can result in higher-quality dielectric film with less traps but pentacene film grown on $\mathrm{HfLaO}$ has been demonstrated to have larger grains. ${ }^{30}$ Therefore, the slope of the LFN for the OTFT with $\mathrm{HfO}_{2}$ gate dielectric is larger. Moreover, the density of traps in the accumulation layer is also in good agreement with those in the literature. ${ }^{12,31,32}$ The extracted density of traps in the $\mathrm{HfO}_{2}$ gate dielectric is also comparable with those reported in Refs. 33-35.

The PSD of drain-current noise for the OTFT with $\mathrm{HfLaO}$ gate dielectric is measured at various drain biases and gate voltages and shown in Fig. 4. As we move from the lowest curve to the highest curve, the magnitude of the drain current increases (see the legends in Fig. 4). Therefore, the magnitude of the PSD scales with the drain current in the channel, as predicted by Eq. (5) of the proposed model.

\section{CONCLUSION}

Based on the GR process in organic semiconductor and gate dielectric, we have developed a microscopic model which provides a quantitative interpretation of the $\mathrm{LFN}$ for the pentacene OTFTs. The model reveals the relation between the density of traps in the pentacene accumulation layer and the slope of the PSD, and fits the measured noise spectrum well in both its slope and magnitude. The density of traps extracted by this model in the accumulation layer is much higher than that in the gate dielectric, and thus dominates the low-frequency noise of the pentacene OTFTs. In conclusion, this model can well explain the LFN measured for the pentacene OTFTs.

\section{ACKNOWLEDGMENTS}

The authors would like to thank Dickey Ma and Linfeng Deng for their help and assistance. This work is supported by the URC for Seed Fund for Strategic Research Theme of $\mathrm{HKU}$ on Molecular Materials, and the University Development Fund (Nanotechnology Research Institute, 00600009) of the University of Hong Kong.

\section{APPENDIX A: PROOF OF PSD IN ELEMENTAL VOLUMES}

The carrier number $\Delta N(t)$ in the volume $\Delta \Lambda$ and $\Delta \Lambda^{\prime}$ at the point $x$ along the channel can be written as: ${ }^{36}$

$$
\Delta N(t)=\overline{\Delta N}+\delta N(t),
$$

where $\overline{\Delta N}$ is the mean value or steady-state value of the carrier number, and $\delta N(t)$ is the fluctuation in carrier number in the volume $\Delta \Lambda$ and $\Delta \Lambda^{\prime}$ caused by the traps in the gate dielectric and accumulation layer.

Since the fluctuations in carrier number are much smaller than the carrier number in the channel, the impact of the fluctuations in carrier number can be linearized as ${ }^{37}$

$$
\delta N=\delta N_{d}+\delta N_{b},
$$

where $\delta N_{d}$ and $\delta N_{b}$ are fluctuations in carrier number caused by traps in the gate dielectric and accumulation layer in the elemental volumes $\Delta \Lambda$ and $\Delta \Lambda^{\prime}$, respectively.

Assuming that (1) $\delta N_{d}$ and $\delta N_{b}$ are due to wide-sense stationary processes with time-averaged value equal to zero, i.e., $\left\langle\delta N_{d}\right\rangle=0$ and $\left\langle\delta N_{b}\right\rangle=0$; (2) the two random processes are mutually independent because they separately occur in the accumulation layer and gate dielectric,

$$
\left\langle(\delta N)^{2}\right\rangle=\left\langle\left(\delta N_{d}\right)^{2}\right\rangle+\left\langle\left(\delta N_{b}\right)^{2}\right\rangle
$$

where \langle\rangle denotes the time-averaged value.

Assuming that all the current in the channel can flow to the drain terminal, the mean-squared fluctuation in current at a distance $x$ along the channel in the volume $\Delta \Lambda$ and $\Delta \Lambda^{\prime}$ can be given by ${ }^{37}$

$$
\left\langle\left(\delta I_{D}\right)^{2}\right\rangle=\left(\frac{I_{D}}{N}\right)^{2}\left\langle(\delta N)^{2}\right\rangle,
$$

where $I_{D}$ is the current in the channel and $N$ is the total carrier number in the channel. Therefore, the PSD of $\left\langle\left(\delta I_{D}\right)^{2}\right\rangle$ is

$$
S_{\delta I_{D}}(f)=\left(\frac{I_{D}}{N}\right)^{2}\left[S_{\delta N_{d}}(f)+S_{\delta N_{b}}(f)\right],
$$

where $S_{\delta N_{d}}(f)$ and $S_{\delta N_{b}}(f)$ are the frequency spectra of $\left\langle\left(\delta N_{d}\right)^{2}\right\rangle$ and $\left\langle\left(\delta N_{b}\right)^{2}\right\rangle$, respectively.

In the elemental volumes $\Delta \Lambda$ and $\Delta \Lambda^{\prime}$, the mean-square fluctuations in carrier number caused by traps in the gate dielectric and in the accumulation layer are, respectively, given by 


$$
\begin{aligned}
& \left\langle\left(\delta N_{d}\right)^{2}\right\rangle=\left\langle\left(\Delta \Lambda^{\prime} \delta \mathrm{n}_{t d}\right)^{2}\right\rangle, \\
& \left\langle\left(\delta N_{b}\right)^{2}\right\rangle=\left\langle\left(\Delta \Lambda \delta \mathrm{n}_{t b}\right)^{2}\right\rangle .
\end{aligned}
$$

From the simple MOSFET theory, it can be shown that

$$
I_{D}=q p(x) \mu \frac{d V(x)}{d x}
$$

where $q$ is the electron charge, $\mu$ is the carrier mobility, $V(x)$ is the electric potential along the channel, and $p(x)$ is the carrier density per unit length in the channel at the point $x$.
The total carrier number $N$ in the channel can be given by

$$
N=\frac{W L C_{o x}}{q}\left|V_{G}-V_{t h}\right|,
$$

where $C_{o x}$ is the oxide capacitance per unit area, $L$ is the channel length, $V_{G}$ is the gate voltage, and $V_{t h}$ is the threshold voltage of the OTFT.

In the volume $\Delta \Lambda$ and $\Delta \Lambda^{\prime}$, the PSD of the meansquared fluctuation in current, $\left\langle\left(\delta I_{D}\right)^{2}\right\rangle$, at the point $x$ along the channel can be derived by using Eqs. (1), (2), and (A4)-(A8) as

$$
S_{\delta I_{D}}(f)=\frac{4 \mu q^{2} I_{D}}{C_{o x} L^{2}\left|V_{G}-V_{t h}\right|}\left[\frac{\tau_{d}}{1+(2 \pi f)^{2} \tau_{d}^{2}} N_{t d} f_{t d} f_{t d p} d y d V+\frac{\tau_{b}}{1+(2 \pi f)^{2} \tau_{b}^{2}} N_{t b} f_{t b} f_{t b p} d y^{\prime} d V\right] .
$$

\section{APPENDIX B: INTEGRAL OF PSD}

$$
\begin{aligned}
S_{I_{D}}(f) & =\frac{4 \mu q^{2} I_{D}}{C_{o x} L^{2}\left|V_{G}-V_{t h}\right|}\left[\iint_{0}^{V_{d} E_{c} t_{d}} \int_{E_{v}} \frac{\tau_{d}(E, V, y)}{1+(2 \pi f)^{2} \tau_{d}^{2}(E, V, y)} N_{t d} f_{t d} f_{t d p} d y d E d V+\int_{0}^{V_{d} E_{c} t_{b}} \int_{E_{v} 0} \frac{\tau_{b}\left(E, V, y^{\prime}\right)}{1+(2 \pi f)^{2} \tau_{b}^{2}\left(E, V, y^{\prime}\right)} N_{t b} f_{t b} f_{t b p} d y^{\prime} d E d V\right] \\
& =\frac{4 \mu q^{2} I_{D}}{C_{o x} L^{2}\left|V_{G}-V_{t h}\right|}\left[\int_{0}^{V_{d}} d V \int_{E_{v}}^{E_{c}} N_{t d} f_{t d} f_{t d p} d E \int_{0}^{t_{d}} \frac{\tau_{d 0} \exp (\gamma y)}{1+(2 \pi f)^{2} \tau_{d 0}^{2} \exp (\gamma y)} d y+\int_{0}^{t_{d}} d V \int_{E_{v}}^{E_{c}} N_{t b} f_{t b} f_{t b p} d E \int_{0}^{t_{b}} \frac{\tau_{b}}{1+(2 \pi f)^{2} \tau_{b}^{2}} d y^{\prime}\right] .
\end{aligned}
$$

Assuming that the traps are uniformly distributed in the bandgap of pentacene,

$$
\int_{E_{v}}^{E_{c}} N_{t d} f_{t d}\left(1-f_{t d p}\right) d E \approx N_{t d} k T,
$$

and

$$
\int_{E_{v}}^{E_{c}} N_{t b} f_{t b}\left(1-f_{t b p}\right) d E \approx N_{t b} k T .
$$

Since the decay time $\tau_{b}$ in the accumulation layer is constant,

$$
\int_{0}^{t_{b}} \frac{\tau_{b}}{1+(2 \pi f)^{2} \tau_{b}^{2}} d y^{\prime}=\frac{t_{b} \tau_{b}}{1+(2 \pi f)^{2} \tau_{b}^{2}} .
$$

In the gate dielectric, $\tau_{d}$ is given by

$$
\tau_{d}(E, V, y)=\tau_{d 0} \exp (\gamma y)
$$

$$
\begin{aligned}
& \int_{0}^{t_{d}} \frac{\tau_{d 0} \exp (\gamma y)}{1+(2 \pi f)^{2} \tau_{d 0}^{2} \exp (\gamma y)} d y \\
& \quad=\frac{1}{2 \pi f \gamma}\left(\arctan \left[2 \pi f \tau_{d 0} \exp \left(\gamma t_{d}\right)\right]-\arctan \left(2 \pi f \tau_{d 0}\right)\right)
\end{aligned}
$$

Typical value of $\tau_{d 0}$ is about $10^{-8} \mathrm{~s}$ and for the traps in the dielectric distributed over a distance of about $t_{d}=2 \mathrm{~nm}, \gamma t_{d}$ is about $40 .^{24}$ Thus, for the low-frequency region, the first term inside the bracket on the right-hand side can be approximated by $\pi / 2$, while the second term is about zero. Therefore,

$$
\int_{0}^{t_{d}} \frac{\tau_{d 0} \exp (\gamma y)}{1+(2 \pi f)^{2} \tau_{d 0}^{2} \exp (\gamma y)} d y \approx \frac{1}{4 f \gamma}
$$

and $S_{I_{D}}(f)$ can be expressed by

$$
\begin{aligned}
S_{I_{D}}(f)= & \frac{k T V_{d} \mu q^{2} I_{D} N_{t d}}{\gamma C_{o x} L^{2}\left|V_{G}-V_{t h}\right|} \frac{1}{f} \\
& +\frac{4 k T V_{d} \mathrm{t}_{b} \tau_{b} \mu q^{2} I_{D} N_{t b}}{C_{o x} L^{2}\left|V_{G}-V_{t h}\right|} \frac{\tau_{b}}{1+\left(2 \pi f \tau_{b}\right)^{2}} .
\end{aligned}
$$


${ }^{1}$ L. Torsi and A. Dodabalapur, Anal. Chem. 77, 380a (2005).

${ }^{2}$ C. D. Sheraw, L. Zhou, J. R. Huang, D. J. Gundlach, T. N. Jackson, M. G. Kane, I. G. Hill, M. S. Hammond, J. Campi, B. K. Greening, J. Francl, and J. West, Appl. Phys. Lett. 80, 1088 (2002).

${ }^{3}$ W. Xu, C. Guo, and S. W. Rhee, J. Mater. Chem. 22, 6597 (2012).

${ }^{4}$ C. D. Dimitrakopoulos and D. J. Mascaro, IBM J. Res. Dev. 45, 11 (2001).

${ }^{5}$ A. Van del Ziel, Noise in Solid State Devices and Circuits (John Wiley \& Sons, New York, 1986), p. 145.

${ }^{6}$ K. K. Hung, P. K. Ko, C. Hu, and Y. C. Cheng, IEEE Trans. Electron Devices 37, 1323 (1990).

${ }^{7}$ S.-M. Hong, C. H. Park, Y. J. Park, and H. S. Min, IEEE Trans. Electron Devices 57, 1110 (2012).

${ }^{8}$ F. N. Hooge, IEEE Trans. Electron Devices 41, 1926 (1994).

${ }^{9}$ A. Van der Ziel, Fluctuation Phenomena in Semiconductors (Butterworths Scientific Publications, London, 1959).

${ }^{10}$ K. K. Hung, P. K. Ko, C. M. Hu, and Y. C. Cheng, IEEE Trans. Electron Devices 37, 654 (1990).

${ }^{11}$ C. Surya and T. Hsiang, Phys. Rev. B 33, 4898 (1986).

${ }^{12}$ W. L. Kalb and B. Batlogg, Phys. Rev. B 81, 035327 (2010).

${ }^{13}$ L. Ke, S. Bin Dolmanan, L. Shen, C. Vijila, S. J. Chua, R. Q. Png, P. J. Chia, L. L. Chua, and P. K. H. Ho, Appl. Phys. Lett. 93, 153507 (2008).

${ }^{14}$ D. Rigaud, M. Valenza, and J. Rhayem, IEEE Proc.: Circuits Devices Syst. 149, 75 (2002).

${ }^{15}$ Y. Xu, T. Minari, K. Tsukagoshi, J. Chroboczek, F. Balestra, and G. Ghibaudo, Solid-State Electron. 61, 106 (2011).

${ }^{16}$ O. D. Jurchescu, B. H. Hamadani, H. D. Xiong, S. K. Park, S. Subramanian, N. M. Zimmerman, J. E. Anthony, T. N. Jackson, and D. J. Gundlach, Appl. Phys. Lett. 92, 132103 (2008).

${ }^{17}$ L. Ke, S. Bin Dolmanan, C. Vijila, S. J. Chua, Y. H. Han, and T. Mei, IEEE Trans. Electron Devices 57, 385 (2010).

${ }^{18}$ L. K. J. Vandamme, R. Feyaerts, Gy Trefán, and C. Detcheverry, J. Appl. Phys. 91, 719 (2002).

${ }^{19}$ K. Y. Choo, S. V. Muniandy, C. L. Chua, and K. L. Woon, Org. Electron. 13, 1370 (2012).
${ }^{20}$ H. K. Kang, L. Jagannathan, and V. Subramanian, Appl. Phys. Lett. 99, 062106 (2011).

${ }^{21}$ L. Ke, S. Bin Dolmanan, L. Shen, C. Vijila, S. J. Chua, R. Q. Png, P. J. Chia, L. L. Chua, and P. K. H. Ho, J. Appl. Phys. 104, 124502 (2008).

${ }^{22}$ C. T. Sah, Proc. IEEE 52, 795 (1964).

${ }^{23}$ M. Kiguchi, M. Nakayama, K. Fujiwara, K. Ueno, T. Shimada, and K. Saiki, Jpn. J. Appl. Phys. 42, L1408 (2003).

${ }^{24}$ S. Christensson, I. Lundstrom, and C. Svensson, Solid-State Electron. 11, 797 (1968).

${ }^{25}$ H. Yoshida and N. Sato, Phys. Rev. B 77, 235205 (2008).

${ }^{26}$ Z. Celik and T. Y. Hsiang, IEEE Trans. Electron Devices 32, 2797 (1985).

${ }^{27}$ L. F. Deng, Y. R. Liu, H. W. Choi, C. M. Che, and P. T. Lai, IEEE Trans. Device Mater. Reliab. 12, 520 (2012).

${ }^{28}$ V. Coropceanu, J. Cornil, D. A. da Silva, Y. Olivier, R. Silbey, and J. L. Bredas, Chem. Rev. 107, 926 (2007).

${ }^{29}$ Y. J. Lin, H. Y. Tsao, and D. S. Liu, Appl. Phys. Lett. 101, 013302 (2012).

${ }^{30}$ L. F. Deng, Ph.D. dissertation ,(The University of Hong Kong, 2011).

${ }^{31}$ D. V. Lang, X. Chi, T. Siegrist, A. M. Sergent, and A. P. Ramirez, Phys. Rev. Lett. 93, 086802 (2004).

${ }^{32}$ W. Kalb, K. Mattenberger, and B. Batlogg, Phys. Rev. B 78, 035334 (2008).

${ }^{33}$ S. Spiga, F. Driussi, A. Lamperti, G. Congedo, and O. Salicio, Appl. Phys. Express 5, 021102 (2012).

${ }^{34}$ I. S. Park, Y. Choi, W. T. Nichols, and J. Ahn, Appl. Phys. Lett. 98, 102905 (2011).

${ }^{35}$ S. Cimino, A. Padovani, L. Larcher, V. V. Afanasév, H. J. Hwang, Y. G. Lee, M. Jurczac, D. Wouters, B. H. Lee, H. Hwang, and L. Pantisano, Microelectron Eng. 95, 71 (2012).

${ }^{36}$ F. Bonani and G. Ghione, Noise in Semiconductor Devices: Modeling and Simulation (Springer, Berlin, 2001), p. 2.

${ }^{37}$ N. B. Lukyanchikova, in Noise Research in Semiconductor Physics, edited by B. K. Jones (Gordon and Breach Science, Amsterdam, 1996), p. 20. 